\title{
An update on allergen immunotherapy
}

\author{
Author: S Hasan Arshad ${ }^{\mathrm{A}}$
}

\begin{abstract}
Allergen-specific immunotherapy (AIT) has been used to treat allergic diseases for over a century. It is the only treatment that addresses immune dysfunction underlying allergic responses rather than simply treating symptoms or suppressing inflammation. Its safety and efficacy in allergic rhinitis, asthma and insect allergy has been documented in a number of systematic reviews but its cost effectiveness is less clear. AIT reduces symptoms and requirement for medication, while improving quality of life. Further, the effect continues for years after discontinuation of treatment. However, evidence regarding cost effectiveness is limited. During AIT, allergen extracts are administered through a subcutaneous or sublingual route for 3-5 years. Subcutaneous immunotherapy is more effective in inducing long-term remission but systemic reactions do occur occasionally. Sublingual immunotherapy is safer and more convenient for the patient but evidence regarding its long-term efficacy is patchy. AIT is used widely in western Europe and the USA. In the UK, subcutaneous immunotherapy is often used for bee and wasp allergy, while both subcutaneous and sublingual immunotherapies are used for severe allergic rhinitis and occasionally for house dust mite and pet allergies. In conclusion, AIT is safe and effective and should be considered as a treatment option for those with allergic diseases.
\end{abstract}

\section{Introduction}

The use of allergen-specific immunotherapy (AIT) to modify the natural course of established disease is unique to allergy medicine among non-communicable diseases. The aim is to induce tolerance to the causative allergen and as a result induce long-term disease remission. This strategy has been successfully used in allergic rhinitis, asthma, venom and drug allergy and, most recently, food allergy and atopic dermatitis have been the focus of research.

AIT was first used to treat hay fever in 1911 at St Mary's Hospital, London. It became popular in the developed world in the post-world war era. Significant progress has been made in the quality and standardisation of allergen extracts, and evidence has accumulated regarding its efficacy and safety. With these attributes, it is startling to see how underused this treatment remains. In the UK, it is largely confined to specialist

Author: ${ }^{A}$ professor of allergy and clinical immunology and honorary consultant physician, Southampton General Hospital, Southampton, UK centres and offered to a small fraction of the patients who could potentially benefit. There is a lack of awareness of AIT, not only in the general population but also among physicians. The Royal College of Physicians has produced several reports describing allergy as an unmet need. ${ }^{1}$ Subsequently, allergy was approved as a distinct discipline with specialist training, which has resulted in increasing use of AIT.

\section{Methods of administration}

AIT is usually administered as subcutaneous injections (subcutaneous immunotherapy (SCIT)) or sublingual immunotherapy (SLIT).

Although a number of variations in the protocol exist, traditionally SCIT is given at weekly intervals until a maintenance dose is reached (usually 4-6 months) and then at 4-8 weekly intervals for 3-5 years (Table 1 ). The treatment is given by trained personnel in outpatient clinics or day centres, where facilities are available to manage rare occurrences of systemic reactions or anaphylaxis. ${ }^{2}$ SCIT should not be given to those with poorly controlled asthma or significant cardiovascular disease as the risk of adverse reaction is higher. ${ }^{3}$

\section{Key points}

Seasonal allergic rhinitis and venom allergy are the two most common clinical indications for the use of allergen immunotherapy in the UK

It is less commonly used for perennial allergic rhinitis because of house dust mite allergy and only in selected cases of asthma

Systemic allergic reactions to allergen immunotherapy are extremely uncommon in the hands of an expert

Increasing confidence in the safety and efficacy of sublingual immunotherapy has made allergen immunotherapy more feasible

The use of allergen immunotherapy in atopic dermatitis and food allergy is a research focus but as yet not in common clinical use

KEYWORDS: Allergen, anaphylaxis, asthma, immunoglobulin $\mathrm{E}$, house dust mite, immunotherapy, pollen, rhinitis 
Table 1. A sample schedule for subcutaneous immunotherapy

\begin{tabular}{|c|c|c|c|}
\hline Dilution & $\begin{array}{l}\text { Concentration, } \\
\mathrm{SQU}^{*} / \mathrm{mL}\end{array}$ & $\begin{array}{l}\text { Volume, } \\
\mathrm{mL}\end{array}$ & Dosage, SQU* \\
\hline \multirow[t]{3}{*}{$1: 1,000$} & 100 & 0.2 & 20 \\
\hline & & 0.4 & 40 \\
\hline & & 0.8 & 80 \\
\hline \multirow[t]{3}{*}{$1: 100$} & 1,000 & 0.2 & 200 \\
\hline & & 0.4 & 400 \\
\hline & & 0.8 & 800 \\
\hline \multirow[t]{3}{*}{$1: 10$} & 10,000 & 0.2 & 2,000 \\
\hline & & 0.4 & 4,000 \\
\hline & & 0.8 & 8,000 \\
\hline \multirow[t]{6}{*}{$1: 1$} & 100,000 & 0.1 & 10,000 \\
\hline & & 0.2 & 20,000 \\
\hline & & 0.4 & 40,000 \\
\hline & & 0.6 & 60,000 \\
\hline & & 0.8 & 80,000 \\
\hline & & 1.0 & 100,000 \\
\hline Maintenance phase & $3-5$ years & 1.0 & 100,000 \\
\hline
\end{tabular}

*SQU (standard quality units) is used here as a generic term to indicate standardisation of allergen content, but the specific units and the amount of allergen varies depending on the type of allergen and the manufacturer. The conventional schedule for subcutaneous immunotherapy with unmodified allergen extracts consists of a dose build up by means of 1-weekly injections, followed by maintenance dose injections at 4-8 week intervals. Rapid up-dosing is possible with a rush protocol.

SCIT is associated with the use of significant healthcare resources and inconvenience for the patient given the need for multiple hospital visits and the long duration of therapy. Rush or cluster protocols have been developed with the aim of reducing the up-dosing duration from months to weeks or even days and have generally been shown to be safe and effective; however, their use remains limited. ${ }^{4}$

SLIT has become popular since the 1990 s. $^{5}$ Sublingual drops, spray or dissolvable tablets of allergen extract are administered once a day (at home) for 3-5 years. A major advantage is the very low risk of systemic reactions and, as a result, the convenience of SLIT being administered at home and the ability to use this even in young children (Table 2).

Both SCIT and SLIT can cause local or systemic reactions. Swelling and redness at the injection site in SCIT and oral itching and tingling in SLIT are common local adverse effects. Systemic reactions are uncommon. ${ }^{3,6}$ In SCIT, mild to moderate systemic reactions occur in approximately $0.1 \%$, while severe reactions are rare ( 1 in 1 million injections). Systemic reactions in SLIT are extremely rare. ${ }^{7}$

\section{Efficacy}

Most systematic reviews have reached the conclusion that both SCIT and SLIT are effective in improving symptoms, quality of life and need for medication. ${ }^{8}$ A major advantage is that the effect persists for years after completion of treatment.
Table 2. Attributes of subcutaneous and sublingual

\section{allergen immunotherapy}

\section{Subcutaneous immunotherapy}

Convenience Inconvenient for patients

$\begin{array}{ll}\text { Staff time } & \begin{array}{l}\text { Significant demand on } \\ \text { staff time }\end{array}\end{array}$

Safety Local adverse effects are common and occur rarely

Feasibility Require expertise and facilities that may not be readily available

Efficacy

(afficacy

Long-term effects

Cost

Effective in allergic rhinitis, asthma and insect allergy

Long-term remission in disease may occur

Cost of treatment extract is lower, but overall cost, given an increased use of healthcare resources, may be higher systemic reactions
Sublingual immunotherapy

Convenient for patients

Minimum use of healthcare resources

Local adverse effects are common but systemic reactions do not occur

Does not require specific expertise or facilities

Effective in allergic rhinitis and asthma

Long-term effects are uncertain

High cost of treatment extract, but minimal use of healthcare resources
This has been well documented for SCIT to pollen, allergic rhinitis, ${ }^{9}$ house dust mite extract, allergic asthma and rhinitis. ${ }^{10}$ Similar evidence is accumulating for SLIT. ${ }^{11}$ A 2010 study using house dust mite sublingual extract in allergic rhinitis demonstrated remission for 7-8 years. ${ }^{11}$ Topical steroid is an inexpensive, effective and relatively safe treatment for most allergic conditions; hence, the cost-effectiveness of AIT is often questioned. However, despite its higher cost in the short term, most studies indicate significant cost savings and improvement in quality of life in the long term. ${ }^{12}$

Allergic patients are often sensitised to several allergens. However, this does not preclude AIT for a single relevant allergen. For example, a patient could be effectively given AIT using grass pollen extract for severe hay fever, ignoring other allergen sensitisation. ${ }^{13}$ AIT using mixed allergens is also possible but evidence regarding the efficacy of this is less clear. ${ }^{13}$ A number of factors influence efficacy and safety, eg if all allergens are clinically relevant, if they can be mixed in a single solution and whether their potencies are cumulatively safe to administer and yet individually high enough to be effective.

\section{Mechanisms of action}

Although the exact mechanisms remain uncertain, following AIT, inflammatory cells such as mast cells, basophils and eosinophils become unresponsive to allergens. This is mediated through the induction of T-regulatory cells, inhibiting allergy promoting T-helper 2 cells. ${ }^{14}$ Further, B-cells produce IgG4 antibodies, which bind to the allergens without initiating a reaction, thus acting as 'blocking antibodies. ${ }^{14}$ 




Fig 1. Management of allergic conditions.

\section{Indications}

The management of allergic conditions includes avoidance of allergen (if possible), pharmacotherapy and immunotherapy (Fig 1). AIT is commonly used for pollen, insect venom, house dust mite and animal dander allergy. Its use for mould and cockroach allergy is less well documented. Patients are selected based on an allergy-focused history and evidence of sensitisation on skin prick test or blood test (measurement of specific IgE to the suspected allergen). For example, a patient with severe seasonal hay fever symptoms and positive skin test to grass pollen would be a suitable candidate.

\section{Allergic conditions with potential use of AIT}

\section{Allergic rhinitis}

AIT should be considered in patients with moderately severe allergic rhinitis who do not respond adequately to standard pharmacotherapy, such as antihistamines, steroid nasal spray and eye drops. ${ }^{3}$ These patients suffer from severe symptoms and their quality of life and work is so adversely affected that they may have to resort to systemic steroids. Standardised allergen extracts are available for grass and tree pollens, house dust mites and animal dander. Mixes of grass and tree pollens can be used, which are generally effective against common types of pollens in the local environment.

For seasonal allergic rhinitis, SCIT to grass and/or tree pollen can now be administered as four pre-seasonal (January to April) injections at 1-2 week intervals, and there is no need to maintain treatment all year round. ${ }^{15-17}$ Thus, it significantly reduces healthcare cost in terms of repeated administration of injections and it is convenient for the patients.

\section{Asthma}

Recent systematic reviews confirm efficacy of AIT, including a steroid-sparing effect, in allergic asthma. ${ }^{18}$ Although both SCIT and SLIT are effective in reducing symptoms and medication requirement, SCIT is more effective than SLIT. In the UK, AIT is rarely used for asthma, partly because of the risk of adverse reaction with SCIT in uncontrolled asthma and partly because of the lack of evidence for its cost effectiveness given currently available effective treatments for asthma.

\section{Atopic dermatitis}

There is some evidence of efficacy of both SCIT and SLIT using house dust mite extracts in mild-to-moderate atopic dermatitis, achieving better control of disease and reducing objectively defined scores for severity and extent of eczema. ${ }^{19}$ However, current use in the UK and throughout the world remains limited because of the lack of evidence from large randomised controlled trials.

\section{Venom allergy}

Bee and wasp allergy can cause anaphylaxis (and occasionally death). AIT is recommended to prevent further allergic reactions in those who have had a severe reaction, are sensitised and there is risk for further stings, eg in bee keepers or gardeners. A 2012 meta-analysis confirmed its effectiveness in preventing further allergic reactions with improvement in quality of life. ${ }^{20}$ The treatment is usually given using SCIT to the relevant allergen (bee or wasp) for 3-5 years.

\section{Drug allergy}

Drug allergy is an adverse reaction to the drug mediated by an immunological mechanism. The most pragmatic approach is to avoid the drug in question and use an alternative. When this is not possible (eg with insulin), desensitisation is possible to allow safe use.

\section{Food allergy}

AIT for food allergy, such as to cow's milk, egg and peanuts, has been investigated using raw or heat modified proteins. Children often tolerate the food when given in gradually increasing amounts so far as they continue to consume the food on a daily basis. ${ }^{21}$ However, adverse reactions are relatively common and the tendency to react to the food reappears if daily consumption is not maintained. AIT to food remains in the research arena.

\section{Prevention of development of allergy}

AIT may prevent development of new sensitisations. Taking this forward, a small 2015 study administered house dust mite SLIT extract to infants who were at risk of development of 
allergy (because of parental history), but have not yet acquired allergic sensitisation. The treatment prevented development of allergic sensitisation. ${ }^{22}$ This may lead to a new indication of AIT with the potential of reducing increasing prevalence of allergic disease. However, large randomised controlled trials are required.

\section{Future use of AIT}

Research is being conducted to individualise AIT, using recombinant antigen technology, to produce allergen extract against specific proteins to which the patient is allergic, rather than the whole allergen. Another approach is to produce extracts using modified proteins or peptides that may increase safety and efficacy. Use of adjuvants to stimulate the immune system to enhance protective immune response is being developed. These approaches have the potential to reduce length of treatment, while improving safety and efficacy.

\section{Conflicts of interest}

SHA has recently attended an advisory board meeting for Allergy Therapeutics, a manufacturer of allergen immunotherapy products.

\section{References}

1 Lee TH. Allergy: the unmet need. Clin Med 2003;3:303-5.

2 Walker SM, Durham SR, Till SJ et al. Immunotherapy for allergic rhinitis. Clin Exp Allergy 2011;41:1177-200.

3 Cox L, Nelson H, Lockey R et al. Allergen immunotherapy: a practice parameter third update. J Allergy Clin Immunol 2011;127(Suppl 1):S1-55.

4 Tabar AI, Echechipía S, García BE et al. Double-blind comparative study of cluster and conventional immunotherapy schedules with Dermatophagoides pteronyssinus. J Allergy Clin Immunol 2005;116:109-18

5 Canonica GW, Cox L, Pawankar R et al. Sublingual immunotherapy: World Allergy Organization position paper 2013 update. World Allergy Organ J 2014;7:6.

6 Epstein TG, Liss GM, Murphy-Berendts K, Bernstein DI. AAAAI/ ACAAI surveillance study of subcutaneous immunotherapy, years 2008-2012: an update on fatal and nonfatal systemic allergic reactions. J Allergy Clin Immunol Pract 2014;2:161-7.

7 Cox LS, Linnemann DL, Nolte $\mathrm{H}$ et al. Sublingual immunotherapy: a comprehensive review. J Allergy Clin Immunol 2006;117:1021-35.

8 Jutel M, Agache I, Bonini S et al. International consensus on allergy immunotherapy. J Allergy Clin Immunol 2015;136:556-68.
9 Durham SR, Walker SM, Varga E-M et al. Long-Term clinical efficacy of grass-pollen immunotherapy. N Engl J Med 1999;341:468-75.

10 Des Roches A, Paradis L, Knani J et al. Immunotherapy with a standardized Dermatophagoides pteronyssinus extract. V. Duration of the efficacy of immunotherapy after its cessation. Allergy 1996;51:430-3.

11 Marogna M, Spadolini I, Massolo A, Canonica GW, Passalacqua G. Long-lasting effects of sublingual immunotherapy according to its duration: a 15-year prospective study. J Allergy Clin Immunol 2010;126:969-75.

12 Berto P, Frati F, Incorvaia C. Economic studies of immunotherapy: a review. Curr Opin Allergy Clin Immunol 2008;8:585-9.

13 Nelson HS. Multiallergen immunotherapy for allergic rhinitis and asthma. J Allergy Clin Immunol 2009;123:763-9.

14 Shamji MH, Durham SR. Mechanisms of immunotherapy to aeroallergens. Clin Exp Allergy 2011;41:1235-46.

15 Drachenberg KJ, Wheeler AW, Stuebner P, Horak F. A well-tolerated grass pollen-specific allergy vaccine containing a novel adjuvant, monophosphoryl lipid A, reduces allergic symptoms after only four preseasonal injections. Allergy 2001;56:498-505.

16 Gawchik SM, Saccar CL. Pollinex Quattro Tree: allergy vaccine. Expert Opin Biol Ther 2009;9:377-82.

17 Rosewich M, Lee D, Zielen S. Pollinex Quattro: an innovative four injections immunotherapy in allergic rhinitis. Hum Vaccin Immunother 2013;9:1523-31.

18 Abramson MJ, Puy RM, Weiner JM. Injection allergen immunotherapy for asthma. Cochrane Database Syst Rev 2010;(8):CD001186.

19 Bae JM, Choi YY, Park CO, Chung KY, Lee KH. Efficacy of allergenspecific immunotherapy for atopic dermatitis: a systematic review and meta-analysis of randomized controlled trials. J Allergy Clin Immunol 2013;132:110-7.

20 Boyle RJ, Elremeli M, Hockenhull J et al. Venom immunotherapy for preventing allergic reactions to insect stings. Cochrane Database Syst Rev 2012;(10):CD008838.

21 Wood RA. Food allergen immunotherapy: current status and prospects for the future. J Allergy Clin Immunol 2016;137:973-82.

22 Zolkipli Z, Roberts G, Cornelius V et al. Randomized controlled trial of primary prevention of atopy using house dust mite allergen oral immunotherapy in early childhood. J Allergy Clin Immunol 2015;136:1541-7.el-11.

Address for correspondence: Professor S Hasan Arshad, MP 810, South Block, Southampton General Hospital, Tremona Road, Southampton S016 6YD, UK.

Email: sha@soton.ac.uk 